Proceedings of the

International Geometry Center

Vol. 10, no. 1 (2017) pp. 1-21

\title{
Complex hyperbolic triangle groups with 2-fold symmetry
}

\author{
John R. Parker, Li-Jie Sun
}

\begin{abstract}
In this paper we will consider the 2-fold symmetric complex hyperbolic triangle groups generated by three complex reflections through angle $2 \pi / p$ with $p \geqslant 2$. We will mainly concentrate on the groups where some elements are elliptic of finite order. Then we will classify all such groups which are candidates for being discrete. There are only 4 types.
\end{abstract}

\section{INTRODUCTION}

A complex hyperbolic triangle is a triple $\left(C_{1}, C_{2}, C_{3}\right)$ of complex geodesics in $\mathbf{H}_{\mathbb{C}}^{2}$. If each pair of complex geodesics intersects in $\mathbf{H}_{\mathbb{C}}^{2} \cup \partial \mathbf{H}_{\mathbb{C}}^{2}$ and the angles between $C_{k-1}$ and $C_{k}$ for $k=1,2,3$ (the indices are taken mod 3) are $\pi / p_{1}, \pi / p_{2}, \pi / p_{3}$, where $p_{1}, p_{2}, p_{3} \in \mathbb{N} \cup\{\infty\}$, we call the triangle $\left(C_{1}, C_{2}, C_{3}\right)$ a $\left(p_{1}, p_{2}, p_{3}\right)$-triangle. The intersection points of pairs of complex geodesics are called the vertices of the complex hyperbolic triangle. A group $\Gamma$ is called a $\left(p_{1}, p_{2}, p_{3}\right)$-triangle group, if $\Gamma$ is generated by three complex reflections $R_{1}, R_{2}, R_{3}$ fixing sides $C_{1}, C_{2}, C_{3}$ of $\left(p_{1}, p_{2}, p_{3}\right)$-triangle. Note that a complex reflection may have order greater than 2 . In what follows we suppose that $R_{1}, R_{2}$ and $R_{3}$ all have order $p \in \mathbb{Z}$ with $p \geqslant 2$.

Any two real hyperbolic triangle groups with the same intersection angles are conjugate in $\operatorname{Isom}^{+}\left(\mathbf{H}^{2}\right)$, which is the orientation preserving isometry group of real hyperbolic plane, see section 10.6 in [1]. If we consider the groups in $\mathrm{PU}(2,1)=\operatorname{Aut}\left(\mathbf{H}_{\mathbb{C}}^{2}\right)$, we will get the nontrivial deformations. The deformation theory of complex hyperbolic triangle groups was begun

This research is carried out while John Parker was visiting Department of Mathematical and Computing Sciences at Tokyo Institute of Technology. The first author is supported by a JSPS Invitation Fellowship. The second author is supported by Grant-in-Aid Scientific Research (B) No. 15H03619 (Sadayoshi Kojima, Tokyo Institute of Technology) and Grantin-Aid Scientific Research (S) No. 24224002 (Takashi Tsuboi, University of Tokyo). We also thank Martin Deraux for several useful conversations.

2010 Mathematics Subject Classification: 20H10, 22E40, 51M10

Keywords: complex hyperbolic triangle groups, discreteness 
in [3] in which they investigated $\Gamma$ of type $(\infty, \infty, \infty)$ with $p=2$ (complex hyperbolic ideal triangle groups). Since then, there have been many developments referring to other types, such as $[15,6,13]$ among which they mainly gave the necessary conditions of $\Gamma$ to be discrete. Especially Parker and Paupert in [10] and [11] investigated the equilateral triangle group generated by three complex reflections with finite order. These include $D e$ raux's lattice, Livné's lattices, Mostow's lattices. Our starting point is a result given by Thompson [14] where he investigated the non-equilateral triangle groups generated by three complex involutions (that is the order of the reflections is $p=2$ ). He obtained his result using a computer search. Using [11] we see that Thompson's results apply to groups with $p>2$ as well. In what follows we will give the specific case about the triangles group with 2 -fold symmetry and we give a rigorous proof.

We will restrict to the complex hyperbolic triangle groups generated by three complex reflections with finite order $p \geq 2$. Suppose that the polar vector of a complex geodesic $C_{1}$ is $\mathbf{v}_{\mathbf{1}}$ (see Section 2 for a more precise explanation). We consider the complex reflection $R_{1}$ in the complex geodesic $C_{1}$. This map sends $\mathbf{v}_{\mathbf{1}}$ to $e^{i \phi} \mathbf{v}_{\mathbf{1}}$ and acts as the identity on the orthogonal complement of $\mathbf{v}_{1}$, that is on vectors that project to $C_{1}$. We will always restrict to the case where $\phi=2 \pi / p$ and so $R_{1}$ has order $p \geqslant 2$. Then $R_{1}$ is given by the following formula:

$$
R_{1}(\mathbf{z})=\mathbf{z}+\left(e^{i \phi}-1\right) \frac{\left\langle\mathbf{z}, \mathbf{v}_{\mathbf{1}}\right\rangle}{\left\langle\mathbf{v}_{\mathbf{1}}, \mathbf{v}_{\mathbf{1}}\right\rangle} \mathbf{v}_{\mathbf{1}} .
$$

In order to convert $R_{1}$ into a matrix with determinant 1 , we need to multiply the expression in (1.1) by $e^{-i \phi / 3}$. The ambiguity involved in this choice is precisely the ambiguity involved in lifting an isometry in $\mathrm{PU}(2,1)$ to a matrix in $\mathrm{SU}(2,1)$.

Here we recall the terminology for braid relations between group elements (see Section 2.2 of Mostow [8]). Let $G$ be a group and $a, b \in G$. Then $a$ and $b$ satisfy a braid relation of length $l \in \mathbb{Z}_{+}$if

$$
(a b)^{l / 2}=(b a)^{l / 2},
$$

where powers means that the corresponding alternating product of $a$ and $b$ should have $l$ factors. For example, $(a b)^{3 / 2}=a b a,(b a)^{2}=b a b a$. We denote the braid length $l$ by $\operatorname{br}(a, b)$ to be the minimum length of a braid relation satisfied by $a$ and $b$.

We define the $\left(l_{1}, l_{2}, l_{3} ; l_{4}\right)$-triangle groups to be the triangle groups with the following braid relations:

$$
\begin{aligned}
& \operatorname{br}\left(R_{2}, R_{3}\right)=l_{1}, \quad \operatorname{br}\left(R_{1}, R_{3}\right)=l_{2}, \\
& \operatorname{br}\left(R_{1}, R_{2}\right)=l_{3}, \quad \operatorname{br}\left(R_{1}, R_{3}^{-1} R_{2} R_{3}\right)=l_{4},
\end{aligned}
$$


where each $R_{j}$ is of order $p$.

In this paper we aim to list the candidates of discrete triangle groups generated by $R_{1}, R_{2}, R_{3}$ with $l_{1}=l_{2}$ and $l_{3}=l_{4}$ as stated in Theorem 2.4.

\section{The PARAmeter SPACE, TRACES AND MAIN RESUlt}

Firstly we recall some fundamentals about complex hyperbolic 2-space. Please refer to $[4,9]$ for more details about the complex hyperbolic space. Let $\mathbb{C}^{2,1}$ denote the vector space $\mathbb{C}^{3}$ equipped with the Hermitian form

$$
\langle\mathbf{z}, \mathbf{w}\rangle=z_{1} \bar{w}_{1}+z_{2} \bar{w}_{2}-z_{3} \bar{w}_{3}
$$

of signature $(2,1)$, where $\mathbf{z}=\left[z_{1}, z_{2}, z_{3}\right]^{t}$ and $\mathbf{w}=\left[w_{1}, w_{2}, w_{3}\right]^{t}$. The Hermitian form divides $\mathbb{C}^{2,1}$ into three parts $V_{-}, V_{0}$ and $V_{+}$, which are

$$
\begin{aligned}
& V_{-}=\left\{\mathbf{z} \in \mathbb{C}^{2,1} \mid\langle\mathbf{z}, \mathbf{z}\rangle<0\right\}, \\
& V_{0}=\left\{\mathbf{z} \in \mathbb{C}^{2,1} \mid\langle\mathbf{z}, \mathbf{z}\rangle=0\right\}, \\
& V_{+}=\left\{\mathbf{z} \in \mathbb{C}^{2,1} \mid\langle\mathbf{z}, \mathbf{z}\rangle>0\right\} .
\end{aligned}
$$

We denote by $\mathbb{C P}^{2}$ the complex projectivisation of $\mathbb{C}^{2,1}$ and by

$$
\mathbb{P}: \mathbb{C}^{2,1} \backslash\{0\} \rightarrow \mathbb{C P}^{2}
$$

the natural projectivisation map. The complex hyperbolic 2 -space $\mathbf{H}_{\mathbb{C}}^{2}$ is defined as $\mathbb{P}\left(V_{-}\right)$. It is called the standard projective model of the complex hyperbolic space. Correspondingly the boundary of $\mathbf{H}_{\mathbb{C}}^{2}$ is $\partial \mathbf{H}_{\mathbb{C}}^{2}=\mathbb{P}\left(V_{0} \backslash\{0\}\right)$. One can also consider the unit ball model whose boundary is the sphere $\mathbb{S}^{3}$ by taking $z_{3}=1$, which can be simply written as

$$
\left\{\left(z_{1}, z_{2}\right) \in \mathbb{C}^{2}:\left|z_{1}\right|^{2}+\left|z_{2}\right|^{2}<1\right\}
$$

The complex hyperbolic plane $\mathbf{H}_{\mathbb{C}}^{2}$ is a Kähler manifold of constant holomorphic sectional curvature -1 . The holomorphic automorphism group of $\mathbf{H}_{\mathbb{C}}^{2}$ is the projectivisation $\mathrm{PU}(2,1)$ of the group $\mathrm{U}(2,1)$ of complex linear transformations on $\mathbb{C}^{2,1}$, which preserve the Hermitian form. Especially $\mathrm{SU}(2,1)$ is the subgroup of $\mathrm{U}(2,1)$ with the determinant of each element being 1 .

Let $x, y \in \mathbf{H}_{\mathbb{C}}^{2}$ be points corresponding to vectors $\mathbf{x}, \mathbf{y} \in \mathbb{C}^{2,1} \backslash\{0\}$. Then the Bergman metric $\rho$ on $\mathbf{H}_{\mathbb{C}}^{2}$ is given by

$$
\cosh ^{2}\left(\frac{\rho(x, y)}{2}\right)=\frac{\langle\mathbf{x}, \mathbf{y}\rangle\langle\mathbf{y}, \mathbf{x}\rangle}{\langle\mathbf{x}, \mathbf{x}\rangle\langle\mathbf{y}, \mathbf{y}\rangle},
$$

where $\mathbf{x}, \mathbf{y} \in V_{-}$are the lifts of $x, y$ respectively. It is easy to check that this definition is independent of the choice of lifts.

Given two points $x$ and $y$ in $\mathbf{H}_{\mathbb{C}}^{2} \cup \partial \mathbf{H}_{\mathbb{C}}^{2}$, with lifts $\mathbf{x}$ and $\mathbf{y}$ to $\mathbb{C}^{2,1}$ respectively, the complex span of $\mathbf{x}$ and $\mathbf{y}$ projects to a complex line in $\mathbb{C P}^{2}$ passing through $x$ and $y$. The intersection of a complex line with $\mathbf{H}_{\mathbb{C}}^{2}$ 
will be called a complex geodesic $C$ (which is homeomorphic to an open 2-dimensional disk), which can be uniquely determined by a positive vector $\mathbf{v} \in V_{+}$, i.e. $C=\mathbb{P}\left(\left\{\mathbf{z} \in \mathbb{C}^{2,1} \backslash\{0\} \mid\langle\mathbf{z}, \mathbf{v}\rangle=0\right\}\right)$. We call $\mathbf{v}$ a polar vector to $C$. As stated in Section 1 , we will consider $\left(l_{1}, l_{2}, l_{3} ; l_{4}\right)$-triangle groups $\Gamma$ generated by three complex reflections, see (1.1), through angle $\phi$ in three complex geodesics.

Throughout this paper, we assume that $R_{1}, R_{2}, R_{3}$ are three complex reflections in complex geodesics $C_{1}, C_{2}, C_{3}$ respectively. We parameterize the triangle groups generated by $R_{1}, R_{2}, R_{3}$ by three complex numbers $\rho$, $\sigma$ and $\tau$. Up to the action of $\mathrm{PU}(2,1)$, we can parameterize the collection of three pairwise distinct complex lines in $\mathbf{H}_{\mathbb{C}}^{2}$ by four real parameters, see Proposition 1 of [12]. The parameters we choose are $|\rho|,|\sigma|,|\tau|$ and $\arg (\rho \sigma \tau)$. In particular, we can freely choose the argument of two out of the three parameters.

Write $u=e^{i \phi / 3}=e^{2 \pi i / 3 p}$. The group $\Gamma$ has generators given by

$$
R_{1}=\left(\begin{array}{ccc}
u^{2} & \rho & -u \bar{\tau} \\
0 & \bar{u} & 0 \\
0 & 0 & \bar{u}
\end{array}\right), \quad R_{2}=\left(\begin{array}{ccc}
\bar{u} & 0 & 0 \\
-u \bar{\rho} & u^{2} & \sigma \\
0 & 0 & \bar{u}
\end{array}\right), \quad R_{3}=\left(\begin{array}{ccc}
\bar{u} & 0 & 0 \\
0 & \bar{u} & 0 \\
\tau & -u \bar{\sigma} & u^{2}
\end{array}\right)
$$

which preserve the Hermitian form

$$
H=\left(\begin{array}{ccc}
\alpha & \beta_{1} & \bar{\beta}_{3} \\
\bar{\beta}_{1} & \alpha & \beta_{2} \\
\beta_{3} & \bar{\beta}_{2} & \alpha
\end{array}\right)
$$

where $\alpha=\sqrt{2-u^{3}-\bar{u}^{3}}, \beta_{1}=-i \bar{u}^{1 / 2} \rho, \beta_{2}=-i \bar{u}^{1 / 2} \sigma, \beta_{3}=-i \bar{u}^{1 / 2} \tau$ (note that here we take $\left.\bar{u}^{1 / 2}=e^{-\pi i / 3 p}\right)$.

This Hermitian form has signature $(2,1)$ if and only if $\operatorname{det}(H)<0$. That is,

$$
\begin{aligned}
0 & <\alpha\left|\beta_{1}\right|^{2}+\alpha\left|\beta_{2}\right|^{2}+\alpha\left|\beta_{3}\right|^{2}-\alpha^{3}-\beta_{1} \beta_{2} \beta_{3}-\bar{\beta}_{1} \bar{\beta}_{2} \bar{\beta}_{3} \\
& =\alpha^{2}|\rho|^{2}+\alpha^{2}|\sigma|^{2}+\alpha^{2}|\tau|^{2}-\alpha^{3}-i \bar{u}^{3 / 2} \rho \sigma \tau+i u^{3 / 2} \bar{\rho} \bar{\sigma} \bar{\tau} .
\end{aligned}
$$

In terms of these parameters

$$
\begin{aligned}
\operatorname{tr}\left(R_{1} R_{2}\right) & =u\left(2-|\rho|^{2}\right)+\bar{u}^{2}, \\
\operatorname{tr}\left(R_{2} R_{3}\right) & =u\left(2-|\sigma|^{2}\right)+\bar{u}^{2}, \\
\operatorname{tr}\left(R_{1} R_{3}\right) & =u\left(2-|\tau|^{2}\right)+\bar{u}^{2}, \\
\operatorname{tr}\left(R_{1} R_{3}^{-1} R_{2} R_{3}\right) & =u\left(2-|\sigma \tau-\bar{\rho}|^{2}\right)+\bar{u}^{2} .
\end{aligned}
$$

Lemma 2.1. [11, Corollary 2.5] If $|\rho|=2 \cos \zeta$, then the three eigenvalues of $R_{1} R_{2}$ will be $\bar{u}^{2},-u e^{2 i \zeta},-u e^{-2 i \zeta}$. 
Proof. Each point on $C_{1}$ is a $\bar{u}=e^{-i \phi / 3}$ eigenvector of $R_{1}$ and each point on $C_{2}$ is a $\bar{u}=e^{-i \phi / 3}$ eigenvector of $R_{2}$, see (1.1). Therefore if $\mathbf{z} \in C_{1} \cap C_{2}$, then we will get that

$$
R_{1} R_{2}(\mathbf{z})=e^{-i \phi / 3} R_{1}(\mathbf{z})=e^{-2 i \phi / 3} \mathbf{z}
$$

Hence $\mathbf{z}$ is a $\bar{u}^{2}=e^{-2 i \phi 3}$ eigenvector of $R_{1} R_{2}$. Hence the sum of the other two eigenvalues of $R_{1} R_{2}$ is $u\left(2-|\rho|^{2}\right)$. By the assumption $|\rho|=2 \cos \zeta$, we know that $R_{1} R_{2}$ is not loxodromic, see Section 6.2 in [4]. Therefore each eigenvalue of $R_{1} R_{2}$ is of modulus one. Then we can get that the three eigenvalues of $R_{1} R_{2}$ will be $\bar{u}^{2},-u e^{2 i \zeta},-u e^{-2 i \zeta}$ from the form of $\operatorname{tr}\left(R_{1} R_{2}\right)$ in (2.3).

Remark 2.2. We suppose that $m \in \mathbb{N}, m \geqslant 2$. If $|\rho|=2 \cos (\pi / m)$ then $\operatorname{br}\left(R_{1}, R_{2}\right)=m$, see Section 2.2 in [8] for details or more precisely [2, Proposition 2.3]. (In fact this is true if $|\rho|=2 \cos (k \pi / m)$ where $k$ is coprime to $m$.) In the following Theorem 2.4, we suppose

$$
|\rho|=|\sigma \tau-\bar{\rho}|=2 \cos (\pi / m), \quad|\sigma|=|\tau|=2 \cos (\pi / n),
$$

which is the case of interest in [2].

If $R_{1}, R_{2}$ are complex involutions $(p=2)$, then the order of $R_{1} R_{2}$ will be of $m$.

Assume that

$$
\operatorname{br}\left(R_{1}, R_{2}\right)=\operatorname{br}\left(R_{1}, R_{3}^{-1} R_{2} R_{3}\right), \quad \operatorname{br}\left(R_{2}, R_{3}\right)=\operatorname{br}\left(R_{1}, R_{3}\right) .
$$

From Remark 2.2 and (2.3), our hypothesis on braiding implies that

$$
|\rho|=|\sigma \tau-\bar{\rho}|, \quad|\sigma|=|\tau| .
$$

Since we are free to choose the argument of two of the three parameters, we impose the condition that $\sigma$ and $\tau$ should be real and non-negative, which means that $\operatorname{Im}(\rho)=\operatorname{Im}(\sigma \tau-\bar{\rho})$. So the condition $|\rho|=|\sigma \tau-\bar{\rho}|$ becomes either $\sigma \tau=\rho+\bar{\rho}$ or $\sigma \tau=0$. In the latter case the group is reducible, so we do not consider it. Hence we suppose $\operatorname{Re}(\rho)>0$ and $\sigma=\tau=\sqrt{\rho+\bar{\rho}}$.

Suppose that $|\rho|=2 \cos (\pi / m)$ and $\sigma=\tau=2 \cos (\pi / n)$, where $m, n \in \mathbb{N}$ and $m, n \geqslant 3$. Then the matrices in (2.1) become:

$$
\begin{aligned}
& R_{1}=\left(\begin{array}{ccc}
u^{2} & \rho & -u \sqrt{\rho+\bar{\rho}} \\
0 & \bar{u} & 0 \\
0 & 0 & \bar{u}
\end{array}\right), \\
& R_{2}=\left(\begin{array}{ccc}
\bar{u} & 0 & 0 \\
-u \bar{\rho} & u^{2} & \sqrt{\rho+\bar{\rho}} \\
0 & 0 & \bar{u}
\end{array}\right),
\end{aligned}
$$




$$
R_{3}=\left(\begin{array}{ccc}
\bar{u} & 0 & 0 \\
0 & \bar{u} & 0 \\
\sqrt{\rho+\bar{\rho}} & -u \sqrt{\rho+\bar{\rho}} & u^{2}
\end{array}\right) .
$$

Furthermore, the Hermitian form $H(2.2)$ has signature $(2,1)$ if and only if

$$
\begin{aligned}
0 & <\alpha|\rho|^{2}+2 \alpha(\rho+\bar{\rho})-\alpha^{3}-i \bar{u}^{3 / 2}\left(\rho^{2}+|\rho|^{2}\right)+i u^{3 / 2}\left(\bar{\rho}^{2}+|\rho|^{2}\right) \\
& =2 \alpha(\rho+\bar{\rho})-\alpha^{3}-i \bar{u}^{3 / 2} \rho^{2}+i u^{3 / 2} \bar{\rho}^{2} .
\end{aligned}
$$

Proposition 2.3. Let

Then

$$
S=\left(\begin{array}{ccc}
\rho & u(1-\rho-\bar{\rho}) & u^{2} \sqrt{\rho+\bar{\rho}} \\
\bar{u} & 0 & 0 \\
0 & \bar{u} \sqrt{\rho+\bar{\rho}} & -1
\end{array}\right) .
$$

(a) $S^{2}=R_{1} R_{2} R_{3}$,

(b)

$$
\begin{aligned}
S R_{1} S^{-1} & =R_{1} R_{2} R_{1}^{-1} \\
S R_{2} S^{-1} & =R_{1} R_{3} R_{1} R_{3}^{-1} R_{1}^{-1} \\
S R_{3} S^{-1} & =R_{1} R_{3} R_{1}^{-1} .
\end{aligned}
$$

In particular,

$$
S\left(R_{2} R_{3}\right) S^{-1}=R_{1} R_{3}, \quad S\left(R_{1} R_{3}^{-1} R_{2} R_{3}\right) S^{-1}=R_{1} R_{2} .
$$

Moreover, $S$ is the only matrix in $S U(2,1)$ satisfying $(a)$ and $(b)$.

Proof. Suppose that $S$ satisfies $(b)$. The basis vectors $\mathbf{v}_{1}=[1,0,0]^{t}$, $\mathbf{v}_{2}=[0,1,0]^{t}$ and $\mathbf{v}_{3}=[0,0,1]^{t}$ are the polar vectors to the fixed complex geodesics of $R_{1}, R_{2}, R_{3}$ respectively. Since $S R_{1} S^{-1}=R_{1} R_{2} R_{1}^{-1}$, we see that $S$ sends $\mathbf{v}_{1}$ to a vector that is polar to the fixed complex geodesic of $R_{1} R_{2} R_{1}^{-1}$, which is a non-zero multiple of $R_{1} \mathbf{v}_{2}$. Similarly for the other complex reflections. Therefore

$$
S \mathbf{v}_{1}=\lambda R_{1} \mathbf{v}_{2}, \quad S \mathbf{v}_{2}=\mu R_{1} R_{3} \mathbf{v}_{1}, \quad S \mathbf{v}_{3}=\nu R_{1} \mathbf{v}_{3} .
$$

Hence any matrix $S$ satisfying $(b)$ has the form:

$$
S=\left(\begin{array}{ccc}
\lambda \rho & \mu u(1-\rho-\bar{\rho}) & -\nu u \sqrt{\rho+\bar{\rho}} \\
\lambda \bar{u} & 0 & 0 \\
0 & \mu \bar{u} \sqrt{\rho+\bar{\rho}} & \nu \bar{u}
\end{array}\right),
$$

where $\lambda, \mu, \nu \in \mathbb{C}-\{0\}$. Now squaring $S$ and comparing its entries with the entries of $R_{1} R_{2} R_{3}$, we see that if such a matrix $S$ also satisfies $(a)$, then we must have:

$$
\lambda^{2}=1, \quad \lambda \mu=1, \quad \mu \nu=-u, \quad \lambda \nu=-u, \quad \nu^{2}=u^{2} .
$$


Also, since $S \in S U(2,1)$ we have $1=\operatorname{det}(S)=-\lambda \mu \nu \bar{u}$. The only solution to these equations is $\lambda=\mu=1$ and $\nu=-u$. Hence $S$ has the form we claimed.

Finally, it is easy to check directly that the matrix $S$ in the statement of the proposition lies in $\mathrm{SU}(2,1)$ and satisfies $(a)$ and $(b)$.

In the following we will classify all discrete triangle groups generated by $R_{1}, R_{2}, R_{3}$ with the 2 -fold symmetry given by $S$ satisfying the conditions $(a)$ and $(b)$ in Proposition 2.3.

Theorem 2.4. Let $R_{1}, R_{2}, R_{3}$ be three complex reflections of order $p$ (with $p \geqslant 2)$ in $S U(2,1)$ so that $R_{i}$ keeps a complex geodesic $C_{i}(i=1,2,3)$ invariant. Assume that there is $S \in S U(2,1)$ such that

$$
\begin{aligned}
S R_{1} S^{-1} & =R_{1} R_{2} R_{1}^{-1}, \\
S R_{2} S^{-1} & =R_{1} R_{3} R_{1} R_{3}^{-1} R_{1}^{-1}, \\
S R_{3} S^{-1} & =R_{1} R_{3} R_{1}^{-1}, \\
S^{2} & =R_{1} R_{2} R_{3} .
\end{aligned}
$$

Let $\rho$ and $\sigma$ be as in (2.3). Suppose $|\rho|=2 \cos (\pi / m)$ and $|\sigma|=2 \cos (\pi / n)$, which implies that $\operatorname{br}\left(R_{1}, R_{3}\right)=n, \operatorname{br}\left(R_{1}, R_{2}\right)=m$ (where $m, n \in \mathbb{N}$ and $m, n \geqslant 3)$. Suppose also that $R_{1} R_{2} R_{3}$ is of finite order. Then the the possible values for $(n, m)$ are $(3,4),(3,5),(4,3),(5,4),(8,6)$ and $(k, k)$ $(k \in \mathbb{N}$ and $k \geqslant 3)$.

Moreover, in each case the group preserves a Hermitian form $H$. When $(n, m)$ is one of $(3,5)$ or $(k, k)$ for $k \geqslant 5$ the form $H$ has signature $(2,1)$ for all $p \geqslant 2$. For the other values of $(n, m)$ the form $H$ only has signature $(2,1)$ for the following values of $p$ :

$$
\begin{array}{lll}
(3,4), p \geqslant 5 ; & (4,3), p \geqslant 4 ; & (5,4), p \geqslant 3 ; \\
(8,6), p \geqslant 3 ; & (3,3), p \geqslant 4 ; & (4,4), p \geqslant 3 .
\end{array}
$$

Note that the solutions correspond to the following parameter values, or their complex conjugates:

\begin{tabular}{|l||l|l|l|}
\hline$(n, m)$ & $\rho$ & $s=\rho-1$ & $\sigma=\tau$ \\
\hline$(3,4)$ & $(1+i \sqrt{7}) / 2$ & $e^{2 \pi i / 7}+e^{4 \pi i / 7}+e^{-6 \pi i / 7}$ & 1 \\
$(3,5)$ & $2 e^{2 \pi i / 5} \cos (\pi / 5)$ & $e^{2 \pi i / 5}+e^{7 \pi i / 15}+e^{-13 \pi i / 15}$ & 1 \\
$(4,3)$ & 1 & 0 & $\sqrt{2}$ \\
$(5,4)$ & $(1+i \sqrt{3})(\sqrt{5}-i \sqrt{3}) / 4$ & $e^{-2 \pi i / 3}+e^{2 \pi i / 15}+e^{8 \pi i / 15}$ & $(1+\sqrt{5}) / 2$ \\
$(8,6)$ & $(1+i)(1-i / \sqrt{2})$ & $e^{\pi i / 2}+e^{\pi i / 12}+e^{-7 \pi i / 12}$ & $\sqrt{2+\sqrt{2}}$ \\
$(k, k)$ & $2 e^{i \pi / k} \cos (\pi / k)$ & $e^{2 \pi i / k}$ & $2 \cos (\pi / k)$ \\
\hline
\end{tabular}




\section{THE PROOF}

Firstly a direct computation will show that the symmetry $S$ conjugates $R_{1} R_{2}$ to $R_{1} R_{3}^{-1} R_{2} R_{3}$ and conjugates $R_{2} R_{3}$ to $R_{1} R_{3}$. It means that

$$
\operatorname{br}\left(R_{1}, R_{2}\right)=b r\left(R_{1}, R_{3}^{-1} R_{2} R_{3}\right), \quad \operatorname{br}\left(R_{2}, R_{3}\right)=b r\left(R_{1}, R_{3}\right)
$$

by recalling Remark 2.2 and (2.3). By the parameterization of the triangle groups in Section 2 and the assumption in Theorem 2.4, one could get the matrix representation of $H, R_{1}, R_{2}, R_{3}$ as (2.2), (2.4), (2.5), (2.6), where

$$
|\rho|=2 \cos (\pi / m), \quad \sigma=\tau=2 \cos (\pi / n) .
$$

Throughout the proof we let $\zeta=\pi / m$ and $\eta=\pi / n$. Then by Proposition 2.3, we can get the unique matrix from of $S \in \mathrm{SU}(2,1)$.

Because of $S^{2}=R_{1} R_{2} R_{3}$, we can restrict ourselves to $S$, which is elliptic of finite order. Equivalently, there exist $a$ and $b$ that are rational multiples of $\pi$ for which:

$$
\operatorname{tr}(S)=-1+\rho=e^{i a}+e^{i b}+e^{-i(a+b)} .
$$

Observe that there is some ambiguity in the choice of $a$ and $b$. First, we can permute the three terms in this expression, and so permute $\{a, b,-a-b\}$; secondly we can change the sign of all three terms and, finally, since $\operatorname{tr}(S)$ is only defined up to multiplying by a cube root of unity, we can add the same integer multiple of $2 \pi / 3$ to both $a$ and $b$. We will use these operations to simplify things in our calculations below.

We denote $\operatorname{tr}(S)$ by $s$, then get that

$$
\begin{aligned}
|s|^{2}=1+|\rho|^{2}-2 \operatorname{Re}(\rho) & =\left|e^{i a}+e^{i b}+e^{-i(a+b)}\right|^{2}, \\
\operatorname{Re}(s)=-1+\operatorname{Re}(\rho) & =\cos (a)+\cos (b)+\cos (a+b),
\end{aligned}
$$

Recall that

$$
\begin{aligned}
|\rho|^{2} & =4 \cos ^{2} \zeta=2 \cos (2 \zeta)+2 \\
\operatorname{Re}(\rho) & =\frac{\sigma \tau}{2}=2 \cos ^{2} \eta=\cos (2 \eta)+1
\end{aligned}
$$

The above two equations can be simplified to

$$
\begin{aligned}
& 1=\cos (2 \zeta)-\cos (2 \eta)-\cos (a-b)-\cos (a+2 b)-\cos (2 a+b), \\
& 0=\cos (2 \eta)-\cos (a)-\cos (b)-\cos (a+b) .
\end{aligned}
$$

In what follows we will repeatedly use the following result given by A. Monaghan, which generalizes the result of Conway and Jones for vanishing sums of cosines of rational multiples of $\pi$.

Proposition 3.1. [7, Theorem 2.4.3.1] Suppose that we have at most five distinct rational numbers of $\pi$, for which some rational linear combination of 
their cosines is rational but no proper subset has this property. If $\phi \in(0, \pi)$ and all other angles are normalized to lie in $\left(0, \frac{\pi}{2}\right)$, then the appropriate linear combination is proportional to one of the following:

(a) $0=\cos (\phi)+\cos \left(\phi+\frac{2 \pi}{3}\right)+\cos \left(\phi+\frac{4 \pi}{3}\right)$,

(b) $0=\cos (\phi)+\cos \left(\phi \pm \frac{2 \pi}{5}\right)-\cos \left(\phi \pm \frac{2 \pi}{15}\right)+\cos \left(\phi \pm \frac{7 \pi}{15}\right)$,

(c) $0=\cos (\phi)-\cos \left(\phi \pm \frac{\pi}{5}\right)+\cos \left(\phi \pm \frac{\pi}{15}\right)-\cos \left(\phi \pm \frac{4 \pi}{15}\right)$,

(d) $\frac{1}{2}=\cos \left(\frac{\pi}{3}\right)$,

(e) $\frac{1}{2}=\cos \left(\frac{\pi}{5}\right)-\cos \left(\frac{2 \pi}{5}\right)$,

(f) $\frac{1}{2}=\cos \left(\frac{\pi}{5}\right)-\cos \left(\frac{\pi}{15}\right)+\cos \left(\frac{4 \pi}{15}\right)$,

(g) $\frac{1}{2}=-\cos \left(\frac{2 \pi}{5}\right)+\cos \left(\frac{2 \pi}{15}\right)-\cos \left(\frac{7 \pi}{15}\right)$,

(h) $\frac{1}{2}=-\cos \left(\frac{\pi}{15}\right)+\cos \left(\frac{2 \pi}{15}\right)+\cos \left(\frac{4 \pi}{15}\right)-\cos \left(\frac{7 \pi}{15}\right)$,

(i) $\frac{1}{2}=\cos \left(\frac{\pi}{7}\right)-\cos \left(\frac{2 \pi}{7}\right)+\cos \left(\frac{3 \pi}{7}\right)$,

(j) $\frac{1}{2}=\cos \left(\frac{\pi}{7}\right)-\cos \left(\frac{2 \pi}{7}\right)+\cos \left(\frac{2 \pi}{21}\right)-\cos \left(\frac{5 \pi}{21}\right)$,

(k) $\frac{1}{2}=\cos \left(\frac{\pi}{7}\right)+\cos \left(\frac{3 \pi}{7}\right)-\cos \left(\frac{\pi}{21}\right)+\cos \left(\frac{8 \pi}{21}\right)$,

(l) $\frac{1}{2}=-\cos \left(\frac{2 \pi}{7}\right)+\cos \left(\frac{3 \pi}{7}\right)+\cos \left(\frac{4 \pi}{21}\right)+\cos \left(\frac{10 \pi}{21}\right)$,

(m) $\frac{1}{2}=\cos \left(\frac{\pi}{7}\right)-\cos \left(\frac{\pi}{21}\right)+\cos \left(\frac{2 \pi}{21}\right)-\cos \left(\frac{5 \pi}{21}\right)+\cos \left(\frac{8 \pi}{21}\right)$,

(n) $\frac{1}{2}=-\cos \left(\frac{2 \pi}{7}\right)+\cos \left(\frac{2 \pi}{21}\right)+\cos \left(\frac{4 \pi}{21}\right)-\cos \left(\frac{5 \pi}{21}\right)+\cos \left(\frac{10 \pi}{21}\right)$,

(o) $\frac{1}{2}=\cos \left(\frac{3 \pi}{7}\right)-\cos \left(\frac{\pi}{21}\right)+\cos \left(\frac{4 \pi}{21}\right)+\cos \left(\frac{8 \pi}{21}\right)+\cos \left(\frac{10 \pi}{21}\right)$.

Since the right hand side of equation (3.4) is 1 (rather than 0 or $1 / 2$ ), Monaghan's theorem implies that it must be a sum of (at least) two similar sums involving fewer cosines. We begin by showing that at least one of the cosines must itself be rational.

Proposition 3.2. Suppose that $\zeta=\pi / m, \eta=\pi / n$ and $a, b$ are rational multiples of $\pi$ so that equations (3.4) and (3.5) hold. Then one of the cosines in equation (3.4) must be rational.

Proof. Suppose that none of the cosines are rational. Then (3.4) splits into two rational sums, one of length two and the other of length three, neither of which has a rational subsum. By inspection from Proposition 3.1 we see that these two sums must have the value $0, \pm 1 / 2$. Since they sum to 1 , they must both be $1 / 2$. Therefore, the sum of length 2 must be (e) and the sum of length 3 must be one of (f), (g) or (i).

(1) $1 / 2=\cos (2 \zeta)-\cos (2 \eta)=-\cos (a-b)-\cos (a+2 b)-\cos (2 a+b)$. Since $\zeta=\pi / m$ and $\eta=\pi / n$ the sum (e) implies $2 \zeta=\pi / 5$ and $2 \eta=2 \pi / 5$. 
For the second equation, there are certain symmetry operations on $a$ and $b$ described in the paragraph after equation (3.1) above. Up to these operations, we now list the possible values of $a$ and $b$. In the first column we indicate which of the identities (a) to (o) in Proposition 3.1 we mainly used.

\begin{tabular}{|c|c|c|c|c|c|}
\hline & $a-b$ & $a+2 b$ & $2 a+b$ & $a$ & $b$ \\
\hline (f) & $\pi / 15$ & $11 \pi / 15$ & $4 \pi / 5$ & $13 \pi / 45$ & $2 \pi / 9$ \\
(g) & $2 \pi / 5$ & $7 \pi / 15$ & $13 \pi / 15$ & $19 \pi / 45$ & $\pi / 45$ \\
(i) & $2 \pi / 7$ & $4 \pi / 7$ & $6 \pi / 7$ & $-2 \pi / 7$ & $-4 \pi / 7$ \\
\hline
\end{tabular}

Using $2 \eta=2 \pi / 5$, we see that none of the values in this table satisfy (3.5). Therefore we get no solutions.

(2) $1 / 2=\cos (2 \zeta)-\cos (a-b)=-\cos (2 \eta)-\cos (a+2 b)-\cos (2 a+b)$. The first equation gives $2 \zeta=\pi / 5$ as in case (1) and so $a-b=2 \pi / 5$. Since $a-b=(2 a+b)-(a+2 b)$ the difference of two of the angles in the second equation must be $2 \pi / 5$. By inspection, we see the only solution is $a+2 b=7 \pi / 15$ and $2 a+b=13 \pi / 15$. This means $2 \eta=2 \pi / 5$ and we are back in case $(1)$.

(3) $1 / 2=-\cos (2 \eta)-\cos (a-b)=\cos (2 \zeta)-\cos (a+2 b)-\cos (2 a+b)$. The first equation gives $2 \eta=2 \pi / 5$ as in (1) and so $a-b=4 \pi / 5$. Substituting in the second equation, we see $a+2 b=-\pi / 15$ and $2 a+b=11 \pi / 15$. Thus $2 \zeta=\pi / 5$ and we are back in case (1) again.

(4) $1 / 2=-\cos (a-b)-\cos (a+2 b)=\cos (2 \zeta)-\cos (2 \eta)-\cos (2 a+b)$. Up to symmetries of $a, b$ and $-a-b$, the first sum implies that $a-b=2 \pi / 5$ and $a+2 b=4 \pi / 5$. Hence $2 a+b=6 \pi / 5$ and so the second sum must be (f). Thus $\cos (2 \zeta)=\cos (2 \pi / m)=\cos (4 \pi / 15)$ or $\cos (2 \eta)=$ $\cos (2 \pi / n)=-\cos (4 \pi / 15)$, so either $m$ or $n$ is not an integer. Therefore there are no solutions.

As a consequence of this result, we can consider separate cases where each of the cosines in (3.4) is rational. If either $\cos (2 \zeta)$ or $\cos (2 \eta)$ is rational it must be 0 or $\pm 1 / 2$ since $\zeta=\pi / m$ and $\eta=\pi / n$ where $m$ and $n$ are at least 3 . If one of the other three cosines is rational we can use the allowable symmetries of $a$ and $b$, we to assume that $\cos (a-b)$ is rational. We treat each of these cases separately below. First we eliminate a simple situation which gives us many solutions and will recur in the different cases.

Lemma 3.3. Suppose that $\cos (2 \zeta)=\cos (2 \eta)$, or equivalently $m=n$, then putting $s=e^{ \pm 2 \pi i / m}$ gives a solution to equations (3.4) and (3.5) for all $m \geqslant 3$.

Proof. Substituting $\cos (2 \zeta)=\cos (2 \eta)$ into (3.4) gives:

$$
0=1+\cos (a-b)+\cos (a+2 b)+\cos (2 a+b)
$$




$$
\begin{aligned}
& =2 \cos ^{2}((a-b) / 2)+2 \cos ((a-b) / 2) \cos (3(a+b) / 2) \\
& =4 \cos ((a-b) / 2) \cos ((a+2 b) / 2) \cos ((2 a+b) / 2) .
\end{aligned}
$$

Therefore one of $(a-b),(a+2 b)$ or $(2 a+b)$ is an odd multiple of $\pi$. Without loss of generality, we suppose that $a+2 b=(2 k+1) \pi$. Then we get $-a-b=b-(2 k+1) \pi$ which yields $s=e^{i a}$, where $a$ is a rational multiple of $\pi$. Because $\operatorname{Re}(s)=-1+\operatorname{Re}(\rho)=-1+\frac{|\sigma|^{2}}{2}=\cos (2 \eta)$, we see that $\cos (a)=\cos (2 \pi / m)=\cos (2 \pi / n)$.

Now we consider the signature of the Hermitian form

$$
\operatorname{Det}(H)=i e^{-\frac{4 \theta+3 \phi}{2} i}\left(-1+e^{(2 \theta+\phi) i}\right)\left(e^{i \theta}+e^{i \phi}\right)^{2} .
$$

TABLE 3.1. Signature of Hermitian form

\begin{tabular}{|c|c|c|c|}
\hline$s$ & $(2,1)$ & degenerate & $(3,0)$ \\
\hline$e^{\frac{2 \pi i}{3}}(m=n=3)$ & $p \geqslant 4$ & $p=3$ & $p=2$ \\
\hline$e^{\frac{2 \pi i}{4}}(m=n=4)$ & $p \geqslant 3$ & $p=2$ & none \\
\hline$e^{\frac{2 \pi i}{k}}(m=n=k \geqslant 5)$ & $p \geqslant 2$ & none & none \\
\hline
\end{tabular}

In this case, we get the solution $n=m$.

We now consider the cases where $\cos (2 \zeta), \cos (2 \eta)$ or $\cos (a-b)$ are rational. We will use the following result proved by Parker when he was analyzing the triangle groups with 3 -fold symmetry [10]. In [10] the last two cases were missed out, but this was corrected in [2].

Proposition 3.4. [10, Proposition 3.2] Let $\theta$, $a$ and $b$ be rational multiples of $\pi$. Write $s=e^{i a}+e^{i b}+e^{-i(a+b)}$. Then the only possible solutions to the equation

$$
\cos (2 \theta)-\cos (a-b)-\cos (a+2 b)-\cos (2 a+b)=\frac{1}{2}
$$

give rise to the following values of $\theta$ and $s$, up to changing the sign of $\theta$ and up to conjugating $s$ and multiplying it by a power of $\omega=e^{2 \pi i / 3}$ :

(i) $2 \theta=2 \pi / 3$ and $s=-e^{-i \psi / 3}$ for some angle $\psi$ that is a rational multiple of $\pi$;

(ii) $2 \theta=\psi$ and $s=e^{2 i \psi / 3}+e^{-i \psi / 3}=e^{i \psi / 6} 2 \cos \frac{\psi}{2}$ for some angle $\psi$ that is a rational multiple of $\pi$;

(iii) $2 \theta=\pi / 3$ and $s=e^{i \pi / 3}+e^{-i \pi / 6} 2 \cos \frac{\pi}{4}$;

(iv) $2 \theta=\pi / 5$ and $s=e^{i \pi / 3}+e^{-i \pi / 6} 2 \cos \frac{\pi}{5}$; 
(v) $2 \theta=3 \pi / 5$ and $s=e^{i \pi / 3}+e^{-i \pi / 6} 2 \cos \frac{2 \pi}{5}$;

(vi) $2 \theta=\pi / 2$ and $s=e^{2 \pi i / 7}+e^{4 \pi i / 7}+e^{-6 \pi i / 7}$;

(vii) $2 \theta=\pi / 2$ and $s=e^{2 \pi i / 9}+e^{-i \pi / 9} 2 \cos \frac{2 \pi}{5}$;

(viii) $2 \theta=\pi / 2$ and $s=e^{2 \pi i / 9}+e^{-i \pi / 9} 2 \cos \frac{4 \pi}{5}$;

(ix) $2 \theta=\pi / 7$ and $s=e^{2 \pi i / 9}+e^{-i \pi / 9} 2 \cos \frac{2 \pi}{7}$;

(x) $2 \theta=5 \pi / 7$ and $s=e^{2 \pi i / 9}+e^{-i \pi / 9} 2 \cos \frac{4 \pi}{7}$;

(xi) $2 \theta=3 \pi / 7$ and $s=e^{2 \pi i / 9}+e^{-i \pi / 9} 2 \cos \frac{6 \pi}{7}$;

(xii) $2 \theta=2 \pi / 5$ and $s=1+2 \cos \frac{2 \pi}{5}$;

(xiii) $2 \theta=4 \pi / 5$ and $s=1+2 \cos \frac{4 \pi}{5}$.

Note that for the groups Parker was considering $s=e^{i a}+e^{i b}+e^{-i a-i b}$ was the trace of $R_{1} J$, whereas in our case it is the trace of $S$. In the cases where $\cos (2 \zeta)=1 / 2$ or $\cos (2 \eta)=-1 / 2$ then equation (3.4) reduces to the equation from Proposition 3.4, and we can use that result to find solutions.

Lemma 3.5. Suppose that $\cos (2 \eta)$ is rational. Then the only solutions to (3.4) and (3.5) are $\cos (2 \zeta)=\cos (2 \pi / m)$ and $\cos (2 \eta)=\cos (2 \pi / n)$ where $(n, m)$ is one of $(3,3),(3,4),(3,5),(4,3),(4,4)$ or $(6,6)$.

Proof. Since $\cos (2 \eta)$ is rational and not equal to \pm 1 it can only be 0 or $\pm 1 / 2$. We treat each case separately.

(1) $\cos (2 \eta)=-\frac{1}{2}$, which gives $n=3$. Note that

$$
\frac{1}{2}=\cos (2 \eta)+1=\operatorname{Re}(\rho)=\operatorname{Re}(s)+1
$$

and so $\operatorname{Re}(s)=-1 / 2$. We rewrite (3.4) to give the equation from Proposition 3.4 with $\theta=\zeta$.

By direct calculation, we just need to consider cases (i), (ii) and (vi) because of $\operatorname{Re}(s)=-1 / 2$.

(i) $s=-e^{-i \psi / 3}$ and so $|s|=1$. This yields that $|\rho|=2 \cos (\pi / m)=1$, and so $m=3$. By considering $\operatorname{Re}(s)=-\cos (\theta / 3)$, we know that $\theta= \pm \pi+6 k \pi(k \in \mathbb{Z})$ which means that $s=-e^{\mp i \pi / 3}$. From (2.7), we get that

$$
\operatorname{Det}(H)=\mp \sqrt{3} \cos (\phi / 2)+\sin (\phi / 2)-2 \sin (3 \phi / 2) .
$$

We list the corresponding signature of Hermitian form for different $s$ in Table 3.2. In this case, we get that $n=m=3$. 
TABlE 3.2. Signature of Hermitian form

\begin{tabular}{|c|c|c|c|}
\hline$s$ & $(2,1)$ & degenerate & $(3,0)$ \\
\hline$-e^{-i \pi / 3}$ & $p \geqslant 4$ & $p=3$ & $p=2$ \\
\hline$-e^{i \pi / 3}$ & none & $p=6$ & $p \neq 6$ \\
\hline
\end{tabular}

(ii) $s=e^{2 i \psi / 3}+e^{-i \psi / 3}=e^{i \psi / 6} 2 \cos (\psi / 2)$ where $\psi=2 \theta$. By solving

$$
\begin{aligned}
-1 / 2 & =\operatorname{Re}(s)=\cos (4 \theta / 3)+\cos (2 \theta / 3) \\
& =2 \cos ^{2}(2 \theta / 3)+\cos (2 \theta / 3)-1
\end{aligned}
$$

we obtain $\cos (2 \theta / 3)=(-1 \pm \sqrt{5}) / 4$. That is, $2 \theta / 3= \pm 2 \pi / 5+2 k \pi$ or $\pm 4 \pi / 5+2 k \pi$. Hence $\theta= \pm 3 \pi / 5+3 k \pi$ or $\pm 6 \pi / 5+3 k \pi$. The only solution to $|s|=2|\cos (\theta)|=2 \cos (\pi / m)$ is $m=5$ (coming from $2 \theta / 3=4 \pi / 5-2 \pi)$.

Therefore $s=e^{2 \pi i / 5}+e^{4 \pi i / 5}$ or $s=e^{-2 \pi i / 5}+e^{-4 \pi i / 5}$. In these cases we find, respectively, that:

$$
\begin{aligned}
& \operatorname{Det}(H)=-\sqrt{5+2 \sqrt{5}} \cos \frac{\phi}{2}-(2+\sqrt{5}+4 \cos \phi) \sin \frac{\phi}{2}, \\
& \operatorname{Det}(H)=\sqrt{5+2 \sqrt{5}} \cos \frac{\phi}{2}-(2+\sqrt{5}+4 \cos \phi) \sin \frac{\phi}{2} .
\end{aligned}
$$

TABLE 3.3. Signature of Hermitian form

\begin{tabular}{|c|c|c|c|}
\hline$s$ & $(2,1)$ & degenerate & $(3,0)$ \\
\hline$e^{-\frac{2 \pi i}{5}}+e^{-\frac{4 \pi i}{5}}$ & $p \leqslant 7$ & none & $p \geqslant 8$ \\
\hline$e^{\frac{2 \pi i}{5}}+e^{\frac{4 \pi i}{5}}$ & $p \geqslant 2$ & none & none \\
\hline
\end{tabular}

In this case, we get that $n=3, m=5$.

(vi) Since $s=e^{2 \pi i / 7}+e^{4 \pi i / 7}+e^{-6 \pi i / 7}=(-1+\sqrt{7} i) / 2$, it follows that $\operatorname{Re}(s)=-1 / 2$ and $|s|=\sqrt{2}$ which indicates that $m=4$. A simple calculation yields that

$$
\operatorname{Det}(H)=\frac{1}{2}(1-8 \cos \phi) \sin \frac{\phi}{2}
$$

from which it follows that the signature of the Hermitian form will be of $(2,1)$ for $p \geqslant 5$, otherwise it will be positive. In this case, we get that $n=3, m=4$.

Therefore we obtain the solutions $(n, m)=(3,3),(3,4)$ and $(3,5)$. 
(2) $\cos (2 \eta)=0$. Now we have $|\sigma|^{2}=2$ which yields $\operatorname{Re}(s)=0$. Therefore one can get the following two equations

$$
\left\{\begin{array}{l}
\cos (2 \zeta)-\cos (a-b)-\cos (a+2 b)-\cos (2 a+b)=1 \\
\cos a+\cos b+\cos (a+b)=0
\end{array}\right.
$$

Since the first of these has 1 on the right hand side, it must split as the sum of (at least) two minimal subsums. Treating these case by case we see that the only possibilities are $\cos (2 \zeta)=0$, which yields $m=n=4$ and $\cos (2 \zeta)=-1 / 2$, which gives $n=4$ and $m=3$. The former case is a particular instance of Lemma 3.3. In the latter case we rewrite (3.2) as

$$
|s|^{2}=1+|\rho|^{2}-2 \operatorname{Re}(\rho)=1+2 \cos (2 \zeta)-2 \cos (2 \eta)=0 .
$$

Therefore, the only solution is $s=0$, or equivalently $\rho=1$. This implies that

$$
\operatorname{Det}(H)=-2 \sin \frac{3 \phi}{2}=-2 \sin \frac{3 \pi}{p},
$$

and the signature of the Hermitian form will be positive if $p=2$, degenerate if $p=3$, negative (of signature $(2,1)$ ) if $p \geqslant 4$. Therefore in this case we get that $n=4, m=3$. Hence the only solutions we get in this case are $(n, m)=(4,3)$ and $(4,4)$.

(3) $\cos (2 \eta)=1 / 2$. Now we have $|\sigma|^{2}=3$ from which it follows that $\operatorname{Re}(s)=1 / 2$. We rewrite the two equations

$$
\left\{\begin{array}{l}
\cos (2 \zeta)-\cos (a-b)-\cos (a+2 b)-\cos (2 a+b)=\frac{3}{2}, \\
\cos a+\cos b+\cos (a+b)=\frac{1}{2}
\end{array}\right.
$$

If the second equation is irreducible, then it must be one of Proposition 3.1 parts (f), (g) or enum:posi:1:i. We see in each case that the angles involved do not sum to 0 (making each cosine positive, the sum is $\pi$ times the ratio of two odd integers for each choice of sign). If the second equation splits as the sum of two rational subsums then, without loss of generality, $\cos (a)$ is rational. Hence it is in the set $\{0, \pm 1 / 2 . \pm 1\}$. Simple trigonometry shows that

$$
\begin{aligned}
2 \cos (a / 2) \cos (a / 2+b) & =\cos (b)+\cos (a+b) \\
& =1 / 2-\cos (a) \\
\cos (a+2 b)+1 & =2 \cos ^{2}(a / 2+b) \\
& =(1 / 2-\cos (a))^{2} /(1+\cos (a)) \\
\cos (a-b)+\cos (2 a+b) & =2 \cos (3 a / 2) \cos (a / 2+b)
\end{aligned}
$$




$$
=-2(1 / 2-\cos (a))^{2}
$$

Substituting these identities in the first equation, we see that $\cos (2 \zeta)$ is a rational function of $\cos (a)$, and so is rational. Substituting the different values of $\cos (a)$ gives a solution with $\zeta=\pi / m$ only when $\cos (a)= \pm 1 / 2$. In both cases, $\cos (2 \zeta)=1 / 2$ and so $m=6$. Thus we obtain the solution $(n, m)=(6,6)$.

Lemma 3.6. Suppose that $\cos (2 \zeta)$ is rational. Then the only solutions to (3.4) and (3.5) are $\cos (2 \zeta)=\cos (2 \pi / m)$ and $\cos (2 \eta)=\cos (2 \pi / n)$, where $(n, m)$ is one of $(3,3),(4,3),(4,4),(5,4),(6,6)$ or $(8,6)$.

Proof. Since $\cos (2 \zeta)$ is rational and not equal to \pm 1 it can only be 0 or $\pm 1 / 2$. We treat each case separately.

(1) $\cos (2 \zeta)=1 / 2$, which gives $m=6$. In this case, we know

$$
|s|^{2}=1+2 \cos (2 \zeta)-2 \cos (2 \eta)=2-2 \cos (2 \eta) \text {. }
$$

In this case, we rewrite equation (3.4) to give the equation from Proposition 3.4 with $2 \theta=\pi-2 \eta$. Checking one by one, we will find that there is no value of $s$ in Proposition 3.4 satisfying (3.5) except the cases (i) and (ii). For (i) we have $2 \eta=\pi-2 \theta=\pi / 3$ and so $n=6$ (we have analyzed this case previously). For(ii), we have $\psi=\pi-2 \eta$ and $s=e^{2 i \pi / 3-4 i \eta / 3}+e^{-i \pi / 3+2 i \eta / 3}$. Substituting in equation (3.5) gives

$$
\begin{aligned}
0 & =\cos (2 \eta)-\operatorname{Re}(s) \\
& =-\cos (\pi-2 \eta)-\cos (2 \pi / 3-4 \eta / 3)-\cos (\pi / 3-2 \eta / 3) \\
& =-\cos (2 \pi / 3-4 \eta / 3)(1+2 \cos (\pi / 3-2 \eta / 3)) .
\end{aligned}
$$

The only solution with $\eta=\pi / n$ is when $2 \pi / 3-4 \eta / 3=\pi / 2$. That is, $n=8$. By calculating $\operatorname{Det}(H)=-2 \cos (\phi)(1+2 \sin \phi)$, we see that $H$ is of signature $(3,0)$ for $p=2$ and is of signature $(2,1)$ for any $p \geqslant 3$. In this case, we get $(n, m)=(6,6)$ or $(8,6)$.

(2) $\cos (2 \zeta)=0$, which gives $m=4$. Then we get that $|\rho|^{2}=2$ and $|s|^{2}=3-|\sigma|^{2}$. Also (3.4) can be replaced by

$$
-\cos (2 \eta)-\cos (a-b)-\cos (a+2 b)-\cos (2 a+b)=1 \text {. }
$$

We have already analyzed the case where $\cos (2 \eta)=0$ or $-1 / 2$, which lead to the solution $(n, m)=(3,4)$ or $(4,4)$. If $\cos (2 \eta)=1 / 2$, then $|\sigma|^{2}=3$ induces $s=0$, which contradicts $\operatorname{Re}(s)=-1+|\sigma|^{2} / 2=1 / 2$. Then it suffices for us to consider the following possible values due to $\eta=\pi / n$, 


\begin{tabular}{|c|c|c|c|c|c|c|}
\hline & $2 \eta$ & $a-b$ & $a+2 b$ & $2 a+b$ & $a$ & $b$ \\
\hline (g) & $2 \pi / 5$ & $2 \pi / 3$ & $7 \pi / 15$ & $17 \pi / 15$ & $3 \pi / 5$ & $-\pi / 15$ \\
\hline (e) & $2 \pi / 5$ & $2 \pi / 5$ & $4 \pi / 5$ & $6 \pi / 5$ & $8 \pi / 5$ & $2 \pi / 15$ \\
\hline
\end{tabular}

From this table, we know that $n=5$ and the pair values $a=3 \pi / 5$ and $b=-\pi / 15$ do not satisfy the equation (3.5). However the second line $a=8 \pi / 5$ and $b=2 \pi / 15$ satisfy the equation (3.5) by applying the equation (g) in Proposition 3.1. Then we calculate the signature of the Hermitian form $H$ using (2.7). We see that $H$ is of signature $(3,0)$ for $p=2$ and is of signature $(2,1)$ for any $p \geqslant 3$. In this case, we get that $m=4$ and $n=5$.

(3) $\cos (2 \zeta)=-\frac{1}{2}$. It follows that $m=3$ and

$$
-\cos (2 \eta)-\cos (a-b)-\cos (a+2 b)-\cos (2 a+b)=\frac{3}{2} \text {. }
$$

Also, $\cos (2 \zeta)=-1 / 2$ implies $|\rho|=1$ and so $\cos (2 \eta)+1=\operatorname{Re}(\rho) \leqslant 1$. This means that $\cos (2 \eta) \leq 0$ and so either $\cos (2 \eta)=\cos (2 \pi / n)=-1 / 2$ or 0 . We have analyzed both of these cases already. These give solutions $(n, m)=(3,3)$ or $(4,3)$.

Now we begin to consider the remaining case in which $\cos (a-b)$ is rational.

Lemma 3.7. Suppose that $\cos (a-b)=-1$, then $\cos (2 \zeta)-\cos (2 \eta)=0$, and the possible solutions are given in Lemma 3.3 in which $n=m$.

Proof. It follows from $\cos (a-b)=-1$ that $b=a+(2 k+1) \pi$. Hence we have $\cos (a+2 b)=\cos (3 a)$ and $\cos (2 a+b)=-\cos (3 a)$. Therefore, equation (3.4) reduces to $\cos (2 \zeta)-\cos (2 \eta)=0$, which we have already treated in Lemma 3.3.

Lemma 3.8. Suppose that $\cos (a-b)=-1 / 2, \cos (2 \zeta)$ and $\cos (2 \eta)$ are not rational, $\cos (2 \zeta)-\cos (2 \eta) \neq 0$. Then we get no solutions for $n, m$ such that (3.4) and (3.5) hold.

Proof. It follows from $\cos (a-b)=-1 / 2$ that $b=a \pm 2 \pi / 3+2 k \pi$. Hence we have $\cos (a+2 b)=\cos (3 a \mp 2 \pi / 3)$ and $\cos (2 a+b)=\cos (3 a \pm 2 \pi / 3)$. Therefore equation (3.4) becomes

$$
\begin{aligned}
1 / 2 & =1+\cos (a-b) \\
& =\cos (2 \zeta)-\cos (2 \eta)-\cos (a+2 b)-\cos (2 a+b) \\
& =\cos (2 \zeta)-\cos (2 \eta)+\cos (3 a) .
\end{aligned}
$$

Since we have supposed that $\cos (2 \zeta)$ and $\cos (2 \eta)$ are not rational, the only way this equation can split into to rational subsums is for $\cos (3 a)$ to be 
rational. Investigating the different possibilities, we see that (3.5) then implies $\cos (2 \eta)$ is rational.

Now suppose the equation does not split into two rational sums of cosines. We list the possible values of $2 \zeta, 2 \eta, a, b$ in Table 3.4, up to the allowable symmetries of $a$ and $b$.

However, we note that there are no values of $2 \eta, a, b$ in the list satisfying (3.5). Therefore there are no solutions for $n, m$.

TABLE 3.4.

\begin{tabular}{|c|c|c|c|c|c|c|}
\hline & $2 \zeta$ & $2 \eta$ & $3 a$ & $a-b$ & $a$ & $b$ \\
\hline (e) & $\pi / 5$ & $2 \pi / 5$ & $\pi / 2$ & $2 \pi / 3$ & $\pi / 6$ & $-\pi / 2$ \\
\hline (e) & $2 \pi / 5$ & $\pi / 5$ & 0 & $2 \pi / 3$ & 0 & $-2 \pi / 3$ \\
\hline (f) & $\pi / 5$ & $\pi / 15$ & $4 \pi / 15$ & $2 \pi / 3$ & $4 \pi / 45$ & $-26 \pi / 45$ \\
\hline (g) & $2 \pi / 15$ & $2 \pi / 5$ & $8 \pi / 15$ & $2 \pi / 3$ & $8 \pi / 45$ & $-22 \pi / 45$ \\
\hline (i) & $\pi / 7$ & $2 \pi / 7$ & $3 \pi / 7$ & $2 \pi / 3$ & $\pi / 7$ & $-11 \pi / 21$ \\
\hline
\end{tabular}

Lemma 3.9. Suppose that $\cos (a-b)=0,1 / 2$ or $1, \cos (2 \zeta)$ and $\cos (2 \eta)$ are not rational, $\cos (2 \zeta)-\cos (2 \eta) \neq 0$. Then there are no solutions for $n$, $m$ satisfying both (3.4) and (3.5).

Proof. We immediately get that

$$
\cos (2 \zeta)-\cos (2 \eta)-\cos (a+2 b)-\cos (2 a+b)=1 \text { or } \frac{3}{2} \text { or } 2 .
$$

Since the right hand side is not $0, \pm 1 / 2$, we see that this sum must split into shorter rational sums of cosines. We break down into the following three cases.

(1) Case $\cos (2 \zeta)-\cos (2 \eta)= \pm 1 / 2$.

(i) Suppose $\cos (2 \zeta)-\cos (2 \eta)=1 / 2$. Note that $\zeta=\pi / m$ and $\eta=\pi / n$, where $m, n \in \mathbb{N}$. Therefore we know that $(n, m)$ is $(5,10)$ and

$$
\cos (a-b)+\cos (a+2 b)+\cos (2 a+b)=-\frac{1}{2} .
$$

We have supposed that $\cos (a-b)$ is rational, then using elementary trigonometry arguments, we see that

$$
2 \cos ((a-b) / 2) \cos (3(a+b) / 2)=-\frac{1}{2}-\cos (a-b) .
$$

Squaring both sides and rearranging gives

$$
\cos (3 a+3 b)=\frac{\cos ^{2}(a-b)-3 / 4}{\cos (a-b)+1} .
$$


We have assumed that either $\cos (a-b)=0$ or $\cos (a-b)=1 / 2$ or $\cos (a-b)=1$, which means that $\cos (3 a+3 b)=-3 / 4$ or $-1 / 3$ or $-1 / 8$. It gives a contradiction here.

(ii) $\cos (2 \zeta)-\cos (2 \eta)=-1 / 2$. It follows that

$$
\cos (a+2 b)-\cos (2 a+b)=-\frac{3}{2} \text { or }-2 \text { or }-\frac{5}{2} .
$$

This sum must again split and so both cosines are rational. Therefore the possible values for $\cos (a+2 b)$ are just -1 or $-1 / 2$ which are equivalent to the case where $\cos (a-b)$ is this value, see Lemma 3.7 and Lemma 3.8. However we assumed $\cos (2 \zeta)-\cos (2 \eta) \neq 0$, therefore there are no solutions for $n, m$ satisfying both (3.4) and (3.5).

(2) Assume that $\cos (2 \zeta)-\cos (x)($ or $\cos (2 \eta)+\cos (y))$ is $1 / 2$ or $-1 / 2$, where $x, y \in\{a+2 b, 2 a+b\}$.

Recalling the equation (3.8), $\cos (2 \zeta)-\cos (x)= \pm 1 / 2$ means that $\cos (2 \eta)+\cos (y)$ is one of the values $\{-5 / 2,-2,-3 / 2,-1,-1 / 2\}$. We just need to consider the case $\cos (2 \eta)+\cos (y)=-1 / 2$, because other values of $\cos (2 \eta)+\cos (y)$ mean that $\cos (2 \eta)$ will be rational. Without loss of generality, we suppose that $x=a+2 b, y=2 a+b$ and list the values of $2 \zeta, a+2 b, 2 \eta, 2 a+b$ and corresponding $a-b$ :

\begin{tabular}{|c|c|c|c|c|c|}
\hline & $2 \zeta$ & $a+2 b$ & $2 \eta$ & $2 a+b$ & $a-b$ \\
\hline (e) & $\pi / 5$ & $2 \pi / 5$ & $2 \pi / 5$ & $4 \pi / 5$ & $2 \pi / 5$ \\
\hline (e) & $\pi / 5$ & $-2 \pi / 5$ & $2 \pi / 5$ & $4 \pi / 5$ & $6 \pi / 5$ \\
\hline
\end{tabular}

There are no values of $a-b$ such that $\cos (a-b)=0$ or $\cos (a-b)=1 / 2$ or $\cos (a-b)=1$. Therefore there are no solutions for $n, m$ satisfying both (3.4) and (3.5) in this case.

(3) Suppose that $\cos (x)$ is rational, where $x \in\{a+2 b, 2 a+b\}$. By suitable changes of $a$ and $b$, the cases $\cos (a+2 b)$ or $\cos (2 a+b)$ is $-1 / 2$ or -1 are equivalent to the cases in Lemma 3.7 and Lemma 3.8. Therefore there are no solutions for $n, m$ because we supposed $\cos (2 \zeta)-\cos (2 \eta) \neq 0$.

Then we consider the condition for $\cos (x)$ to be $0,1 / 2$ or 1 and suppose that $x=a+2 b$. We get that

$$
\cos (2 \zeta)-\cos (2 \eta)-\cos (2 a+b) \in\left\{1, \frac{3}{2}, 2, \frac{5}{2}, 3\right\},
$$

which can be reduced to $\cos (2 \zeta)-\cos (2 a+b)$ or $\cos (2 \eta)+\cos (2 a+b)$ is rational which has been considered above.

Now we can get that there are no solutions for $n, m$ satisfying both (3.4) and (3.5) under the conditions in Lemma 3.9. 
We sum up all the possible values for $n, m$ from above process,

Lemma $3.3 n=m \geqslant 3$;

Lemma $3.5 \quad(n, m) \in(3,3),(3,4),(3,5),(4,3),(4,4)$ or $(6,6)$;

Lemma $3.6 \quad(n, m) \in(3,3),(4,3),(4,4),(5,4),(6,6)$ or $(8,6)$;

which we desired. Also we could see the range of $p$ for each possible value to hold from the above analysis.

Remark 3.10. Note that the new candidates for $(n, m)$ to be $(5,4),(4,3)$ and $(8,6)$ do not appear on Thompson's list in [14]. However referring to [2], in what follows we will see that the triangle groups for $(n, m)$ to be $(5,4)$ corresponds to Thompson groups $\mathbf{S}_{2}$ and the triangle groups for $(m, n)$ to be $(4,3)$ is of actually Mostow groups with braiding $(2,3,4 ; 4)$. The pair $(n, m)=(8,6)$ was also found by Deraux when he was making a similar computer search to Thompson (private communication).

Case 1: $(n, m)=(5,4)$. Suppose that $M_{1}, M_{2}, M_{3}$ are three complex reflections of order $p$, which satisfy

$$
\begin{aligned}
& \operatorname{br}\left(M_{1}, M_{2}\right)=4, \\
& \operatorname{br}\left(M_{1}, M_{3}\right)=\operatorname{br}\left(M_{2}, M_{3}\right)=3, \\
& \operatorname{br}\left(M_{1}, M_{3}^{-1} M_{2} M_{3}\right)=5 .
\end{aligned}
$$

Actually, $M_{1}, M_{2}, M_{3}$ will be Thompson group $\mathbf{S}_{2}$. Write $R_{1}=M_{2}^{-1} M_{1} M_{2}$, $R_{2}=M_{1} M_{2} M_{1}^{-1}, R_{3}=M_{3}$. We claim that

$$
\operatorname{br}\left(R_{1}, R_{2}\right)=\operatorname{br}\left(R_{1}, R_{3}^{-1} R_{2} R_{3}\right)=4, \quad \operatorname{br}\left(R_{1}, R_{3}\right)=\operatorname{br}\left(R_{2}, R_{3}\right)=5 .
$$

First, observe, we also have $\operatorname{br}\left(M_{2}^{-1} M_{1} M_{2}, M_{3}\right)=\operatorname{br}\left(M_{1}^{-1} M_{2} M_{1}, M_{3}\right)=5$. Thus

$$
\begin{aligned}
& b r\left(R_{1}, R_{3}\right)=\operatorname{br}\left(M_{2}^{-1} M_{1} M_{2}, M_{3}\right)=5, \\
& \operatorname{br}\left(R_{2}, R_{3}\right)=\operatorname{br}\left(M_{1} M_{2} M_{1}^{-1}, M_{3}\right)=5 .
\end{aligned}
$$

Using $\operatorname{br}\left(M_{1}, M_{2}\right)=4$, we have

$$
R_{1} R_{2}=\left(M_{2}^{-1} M_{1} M_{2}\right)\left(M_{1} M_{2} M_{1}^{-1}\right)=M_{2}^{-1}\left(M_{2} M_{1} M_{2} M_{1}\right) M_{1}^{-1}=M_{1} M_{2} .
$$

Hence $\operatorname{br}\left(R_{1}, R_{2}\right)=\operatorname{br}\left(M_{1}, M_{2}\right)=4$. We denote $M_{1}, M_{1}^{-1}$ by $1, \overline{1}$ simply and so on. Now we consider

$$
\begin{aligned}
R_{1} R_{3}^{-1} R_{2} R_{3} & =M_{2}^{-1} M_{1} M_{2} M_{3}^{-1} M_{1} M_{2} M_{1}^{-1} M_{3} \\
& =\overline{2} 12 \overline{3} 12 \overline{1} 3 \\
& =(123123)(\overline{3} \overline{2} \overline{1} \overline{3} \overline{2} \overline{1} \cdot \overline{2} 12 \overline{3} 12 \overline{1} 3 \cdot 123123) \overline{3} \overline{2} \overline{1} \overline{3} \overline{2} \overline{1} \\
& =(123123)(\overline{3} \overline{2} \overline{1} \overline{3} \cdot 12 \overline{1} \cdot \overline{3} 12 \overline{1} 3 \cdot 123123) \overline{3} \overline{2} \overline{1} \overline{3} \overline{2} \overline{1} \\
& =(123123)(\overline{3} \overline{2}(\overline{1} \overline{3} 1) 2(\overline{1} \overline{3} 1) 2(\overline{1} 31) 23123) \overline{3} \overline{2} \overline{1} \overline{3} \overline{2} \overline{1}
\end{aligned}
$$




$$
\begin{aligned}
& =(123123)(\overline{3} \overline{1} 31 \overline{2} \overline{1} \overline{3} 13123) \overline{3} \overline{2} \overline{1} \overline{3} \overline{2} \overline{1} \\
& =(123123)(1 \overline{3} \overline{2} 323) \overline{3} \overline{2} \overline{1} \overline{3} \overline{2} \overline{1} \\
& =(123123)(12) \overline{3} \overline{2} \overline{1} \overline{3} \overline{2} \overline{1} .
\end{aligned}
$$

Since $R_{1} R_{3}^{-1} R_{2} R_{3}$ is conjugate to $M_{1} M_{2}$ we see that

$$
\operatorname{br}\left(R_{1}, R_{3}^{-1} R_{2} R_{3}\right)=\operatorname{br}\left(M_{1}, M_{2}\right)=4
$$

as claimed. In particular, this shows that this case is equivalent to Thompson groups $\mathbf{S}_{2}$.

Case 2: $(n, m)=(4,3)$. In this case, it is easy to check that $R_{2}, R_{3}$ (also $R_{3}, R_{1}$ ) braid with length $4, R_{1}, R_{2}$ (also $R_{1}, R_{3}^{-1} R_{2} R_{3}$ ) braid with length $3, R_{1}, R_{2} R_{3} R_{2}^{-1}$ (also $R_{3}, R_{1} R_{2} R_{1}^{-1}$ ) braid with length 2 (i.e. they commute) and $R_{1} R_{2} R_{3}$ is regular elliptic of order 3 . Note that $\operatorname{Det}(H)<0$ when $p \geqslant 4$.

As the same fashion in [5], we define $\iota$ by the reflection of group that acts on the generating set $\left(R_{1}, R_{2}, R_{3}\right)$ as follows,

$$
\iota\left(R_{1}\right)=R_{1}, \quad \iota\left(R_{2}\right)=R_{1} R_{2} R_{1}^{-1}, \quad \iota\left(R_{3}\right)=R_{3} .
$$

Under the action of $\iota$, the $(4,4,3 ; 3)$-triangle groups will be sent to the triangle groups with braiding $(2,3,4 ; 4)$

$$
\begin{aligned}
\left\langle\iota\left(R_{1}\right), \iota\left(R_{2}\right) \iota\left(R_{3}\right):\right. & \iota\left(R_{2} R_{3}\right)=\iota\left(R_{3} R_{2}\right),\left(\iota\left(R_{1} R_{2}\right)\right)^{\frac{3}{2}}=\left(\iota\left(R_{2} R_{1}\right)\right)^{\frac{3}{2}}, \\
& \left(\iota\left(R_{1} R_{3}\right)\right)^{2}=\left(\iota\left(R_{3} R_{1}\right)\right)^{2}, \\
& \left.\left(\iota\left(R_{1} R_{2} R_{3} R_{2}^{-1}\right)\right)^{2}=\left(\iota\left(R_{2} R_{3} R_{2}^{-1} R_{1}\right)\right)^{2}\right\rangle .
\end{aligned}
$$

Recall the Mostow groups $\Gamma(p, t)$ mentioned in [8, 10]. For Mostow groups, there exists a complex hyperbolic isometry $J$ of order 3 so that $R_{j+1}=J R_{j} J^{-1}$ and $R_{i} R_{i+1} R_{i}=R_{i+1} R_{i} R_{i+1}$. We could rewrite them as triangle groups with braiding $(2,3,4 ; 4)$ as follows

$$
\begin{aligned}
\left\langle R_{1}, R_{2}, J\left(R_{1} R_{2}\right)^{-1}:\right. & R_{2} J\left(R_{1} R_{2}\right)^{-1}=J\left(R_{1} R_{2}\right)^{-1} R_{2},\left(R_{1} R_{2}\right)^{\frac{3}{2}}=\left(R_{2} R_{1}\right)^{\frac{3}{2}}, \\
& \left(R_{1} J\left(R_{1} R_{2}\right)^{-1}\right)^{2}=\left(J\left(R_{1} R_{2}\right)^{-1} R_{1}\right)^{2} \\
& \left.\left(R_{1} R_{2} J\left(R_{1} R_{2}\right)^{-1} R_{2}^{-1}\right)^{2}=\left(R_{2} J\left(R_{1} R_{2}\right)^{-1} R_{2}^{-1} R_{1}\right)^{2}\right\rangle .
\end{aligned}
$$

\section{REREFENCES}

[1] Alan F. Beardon. The geometry of discrete groups. Springer, New York, 1983.

[2] M. Deraux, J. Parker, J. Paupert. On commensurability classes of non-arithmetic complex hyperbolic lattices. arXiv:1611.00330.

[3] William M. Goldman, John R. Parker. Complex hyperbolic ideal triangle groups. J. Reine Angew. Math., 425:71-86, 1992.

[4] William Mark Goldman. Complex hyperbolic geometry. Oxford University Press, 1999. 
[5] Shigeyasu Kamiya, John R. Parker, James M. Thompson. Notes on complex hyperbolic triangle groups. Conform. Geom. Dyn., 14:202-218, 2010.

[6] Shigeyasu Kamiya, John R. Parker, James M. Thompson. Non-discrete complex hyperbolic triangle groups of type $(n, n, \infty ; k)$. Canad. Math. Bull., 55(2):329-338, 2012.

[7] Andrew Monaghan. Complex hyperbolic triangle groups. Doctoral thesis, 2013.

[8] G. D. Mostow. On a remarkable class of polyhedra in complex hyperbolic space. Pacific J. Math., 86(1):171-276, 1980.

[9] John R. Parker. Complex hyperbolic kleinian groups. Preprint.

[10] John R. Parker. Unfaithful complex hyperbolic triangle groups. I. Involutions. Pacific J. Math., 238(1):145-169, 2008.

[11] John R. Parker, Julien Paupert. Unfaithful complex hyperbolic triangle groups. II. Higher order reflections. Pacific J. Math., 239(2):357-389, 2009.

[12] Anna Pratoussevitch. Traces in complex hyperbolic triangle groups. Geom. Dedicata, 111:159-185, 2005.

[13] Li-Jie Sun. Notes on complex hyperbolic triangle groups of type $(m, n, \infty)$. To appear in Advances in Geometry.

[14] James M. Thompson. Complex hyperbolic triangle groups. Doctoral thesis, 2010.

[15] Justin Olav Wyss-Gallifent. Complex hyperbolic triangle groups. ProQuest LLC, Ann Arbor, MI, 2000. Thesis (Ph.D.) - University of Maryland, College Park.

Received: January 21, 2017, accepted: April 15, 2017.

John R. Parker

University of Durham, South RoAd, Durham DH1 3LE UK

Email: j.r.parker@durham.ac.uk

Li-Jie Sun

Tokyo Institute of Technology, 2-12-1 O-Okayama Meguro-ku, Tokyo 152-8552

JAPAN

Email: lijie.alice.sun@gmail.com 